\title{
CAPACIDADE ADSORTIVA DO CARVÃO ATIVADO DE COCO DE BABAÇU FRENTE À REMOÇÃO DE DIURON EM SOLUÇÃO SINTÉTICA
}

\author{
M. T. WANDEMBRUCK ${ }^{1}{ }^{*}$, D. MANTOVANI ${ }^{1,}$ J. F. HONORIO $^{1}$, R. \\ BERGAMASCO $^{1}$, A. M. S. VIEIRA ${ }^{2}$, M. F. VIEIRA ${ }^{1}$ \\ ${ }^{1}$ Universidade Estadual de Maringá, Departamento de Engenharia Química \\ ${ }^{2}$ Universidade Estadual de Maringá, Departamento de Engenharia de Alimentos \\ *e-mail: mari_wandembruck@ hotmail.com
}

\begin{abstract}
RESUMO
A agricultura é considerada uma das principais atividades que contribuem para a contaminação ambiental, pois utiliza um alto consumo de herbicidas que apresentam baixa degradação no meio ambiente, o que leva a um maior risco à qualidade de sistemas hídricos. $\mathrm{O}$ diuron destaca-se como um dos principais herbicidas utilizados na produção de cana-de-açúcar. $\mathrm{O}$ presente estudo propõe o tratamento do herbicida diuron por adsorção utilizando carvão ativado granular (CAG) de coco de babaçu como adsorvente. Estudos cinéticos apresentaram tempo de contato de 30 minutos para atingir o equilíbrio de adsorção, sendo bem representados pelo modelo de pseudosegunda ordem. Os dados de isoterma foram bem representados pelo modelo de Freundlich, para as três temperaturas estudadas, apresentando em $45^{\circ} \mathrm{C} \mathrm{q}_{\mathrm{máx}}=41,432 \mathrm{mg} \mathrm{g}^{-1}$. O carvão ativado demonstrou ser promissor na remoção do diuron, apresentando curto tempo de contato e boa capacidade de adsorção.
\end{abstract}

\section{INTRODUÇÃO}

O impacto ambiental dos sistemas hídricos está relacionado a uma série de substâncias tóxicas, como agrotóxicos, fertilizantes, metais pesados, compostos orgânicos voláteis, entre outros, provindos das mais diversas formas de agricultura e industrialização (NETO \& SARCINELLI, 2009).

Brito et al. (2001) e Grutzmacher et al. (2008) relatam que grande parte da contaminação ambiental brasileira provém da agricultura, principalmente quando esta se encontra nas proximidades de áreas de mananciais, aliado a um alto consumo de agrotóxicos. Desde 2008 o Brasil é considerado o principal mercado consumidor de agrotóxicos do mundo (TERRA e PELAEZ, 2008).

O uso de agrotóxicos acima do permitido perante às legislações está diretamente vinculado às plantações de monoculturas de soja, milho, cana-de-açúcar, arroz e algodão que correspondem às principais commodities do agronegócio (SINDIVEG, 2014). Atualmente, esta prática consiste em uma das maiores preocupações no quesito ambiental no que diz respeito à escala local, regional, nacional e global (KONSTANTINOU et al., 2006).

Estas práticas consistem em uma das maiores preocupações no quesito ambiental no que diz respeito à escala local, regional, 
nacional e global (KONSTANTINOU et al., 2006), o qual corrobora para que o Brasil venha sendo considerado o principal mercado consumidor de agrotóxicos do mundo, desde 2008 (TERRA e PELAEZ, 2008).

O cultivo da cana-de-açúcar exige uma constante utilização de herbicidas, a fim de combater a presença de variadas plantas daninhas, o qual está intimamente relacionado à crescente produção nacional desta cultura, concentrada na região Centro-Sul, na zona de recarga do aquífero Guarani (DI BERNARDO DANTAS et al., 2011).

Os herbicidas de maior aplicação e recomendação na produção de cana-de-açúcar são o diuron, metribuzin, halosulfuron, clomazone, ametrina, 2,4-D, entre outros (SILVA, 2004). Esses organoclorados apresentam baixa degradação no solo, ou seja, são altamente persistentes no ambiente (SANCHES et al., 2003). Além disso, atuam com potencialidades toxinogênicas, carcinogênicas, mutagênicas, entre outras; (ZOLGHARNEIN et al., 2011).

O diuron ou 3-(3,4-diclorofenil)-1,1dimetilureia, pertencente à família das feniluréias é considerado particularmente polar e hidrofóbico, não ionizável, potencialmente tóxico ao meio ambiente (classe II), com solubilidade baixa de $42 \mathrm{mg}$ $\mathrm{L}^{-1}$ em pH 7,0 $\left(25^{\circ} \mathrm{C}\right)$ e tempo de meia-vida no solo de 112 a 134 dias (LAVORENTI e LANÇAS, 2003). Sua principal ação ocorre em pré e pós emergência (germinação) de monoculturas de cana-de-açúcar, citros, café, algodão, entre outros.

Visando a remoção de matrizes orgânicas e inorgânicas, inclusive herbicidas como o diuron, vários processos tecnológicos vêm sendo utilizados para tratamento, como a oxidação química, adsorção, sedimentação e filtração (DI BERNARDO e DANTAS, 2005; SHWARZENBACH et al., 2006). Entretanto, a adsorção tem-se destacado por consistir em um projeto simples, de fácil operação, baixo custo, flexibilidade, entre outros, apresentando bons resultados de forma rápida na remoção de poluentes (BHATNAGAR e SILLANPÄ̈̈, 2010).

$\mathrm{O}$ setor industrial brasileiro gera muitos subprodutos, os quais podem ser reaproveitados comercialmente, como o uso de adsorventes provindos de cascas de coco, babaçu, dendê, mamona, arroz, trigo, café, soja, entre outros. Sobretudo, vários estudos utilizam carvão ativado desses materiais vegetais na adsorção, para remoção de agrotóxicos no tratamento de águas e efluentes industriais (PIZA, 2008; RUEDA, 2008).

Neste contexto, o presente estudo teve como objetivo a remoção de diuron por adsorção em carvão ativado de coco de babaçu em águas sintéticas.

\section{METODOLOGIA}

O material adsorvente utilizado foi o carvão granular de coco de babaçu (CAG), ativado em altas temperaturas $\left(350-450^{\circ} \mathrm{C}\right)$, para melhoramento do processo adsortivo. $\mathrm{O}$ CAG foi fornecido pela empresa Tobasa Tocantins Babaçu S/A, da região de Cinelandia/RJ.

\subsection{Preparo das soluções}

A curva de calibração foi obtida utilizando diuron (Teor: 99,8\%), da Sigma Aldrich. Uma solução padrão de diuron foi preparada na concentração de $1000 \mathrm{mg} \mathrm{L}^{-1}$ separadamente, pesando $0,01 \mathrm{~g}$ de analito e dissolvendo em $10 \mathrm{ml}$ de álcool metílico (Teor: 99,8\%) grau HPLC, sendo armazenadas no freezer. A partir desta solução padrão a curva de calibração foi obtida diluindo amostras em água ultrapura Milli-Q para as concentrações de 20 a 0,0125 $\mathrm{mg} \mathrm{L}^{-1}$.

As soluções utilizadas nos ensaios de adsorção foram preparadas a partir do diuron 
Nortox 500 SC, constituinte de $50 \%$ de diuron. As soluções foram preparadas utilizado água ultrapura Milli-Q contaminada com esse diuron na concentração de $20 \mathrm{mgL}^{-1}$.

\subsection{Técnica analítica}

As leituras foram realizadas por Cromatografia Líquida de Alta Eficiência (CLAE), marca Gilson, controlado pelo Software Born. O equipamento possui injetor manual Rheodyne, modelo 307 e dectetor UV/Visível, modelo 151. A análise foi conduzida utilizando como fase móvel acetonitrila/água ultrapura do sistema Millipore ${ }^{\circledR}$ Milli-Q, na relação (65/35) (v/v) e coluna (C18 Microsorb $5 \mu \mathrm{m}, 100 \AA$ A, 4,6x250 $\mathrm{mm})$, com vazão de $0,75 \mathrm{~mL} \mathrm{~min}^{-1}$, volume de injeção de $20 \mu \mathrm{L}$, em bomba binária, e leituras em comprimento de onda de $247 \mathrm{~nm}$.

\subsection{Caracterização do adsorvente}

\subsubsection{Caracterização do ponto de carga zero (PCZ)}

O ponto de carga zero é definido como o pH em que a superfície do sólido possui carga neutra (REGALBUTO e ROBLES, 2004). A metodologia empregada neste trabalho é denominada "experimento dos 11 pontos" (REGALBUTO e ROBLES, 2004). O procedimento consistiu na mistura de $50 \mathrm{mg}$ do adsorvente em $50 \mathrm{~mL}$ de água deionizada sob 11 diferentes condições de $\mathrm{pH}$ inicial, com adaptações, entre eles: 1, 2, 3, 4, 5, 6, 8, 9, 10,11 e 12 com ajuste de $\mathrm{HCl}$ e $\mathrm{NaOH} 0,1$ M e $1 \mathrm{M}$, em agitação de $200 \mathrm{rpm}$ com medição do $\mathrm{pH}$ após $4 \mathrm{~h}$ (REGALBUTO e ROBLES, 2004; GUILARDUCI et al., 2006; MIMURA et al., 2010). Os resultados foram apresentados relacionando o $\mathrm{pH}$ final versus $\mathrm{pH}$ inicial. $\mathrm{O}$ valor referente ao $\mathrm{PCZ}$ corresponde à faixa na qual o $\mathrm{pH}$ final mantiver constante independente do $\mathrm{pH}$ inicial.
2.3.2 Caracterização da área superficial específica (BET)

$\mathrm{Na}$ caracterização textural do CAG analisou-se a área superficial específica pelo método BET (BRUNAUER et al., 1938) a partir de isotermas de adsorção usando o gás $\mathrm{N}_{2}$ a $77 \mathrm{~K}$, na faixa de pressão $\mathrm{P} / \mathrm{P}_{0}$ entre 0,05 e 0,35 . O equipamento utilizado foi o NOVA 1000 Séries com analisador da QuantaChrome.

A partir desta caracterização, calcularam-se os seguintes parâmetros: a área e o volume de microporos (método $\mathrm{t}$ de Halsey), o diâmetro de microporos (método DA) e a área, o volume e o diâmetro de mesoporos (método BJH).

\subsection{Ensaios de adsorção}

Foram realizados ensaios cinéticos e isotermas para obtenção do tempo de contato, bem como estimativa da capacidade máxima de adsorção do CAG de coco de babaçu aplicando modelos apresentados na literatura.

Os respectivos experimentos foram realizados no Laboratório de Gestão, Controle e Preservação Ambiental (LGCPA), do Departamento de Engenharia Química (DEQ), da Universidade Estadual de Maringá (UEM), Maringá, Paraná, Brasil.

\subsubsection{Cinética de adsorção}

Os ensaios referentes a cinética de adsorção foram realizados com 0,5 gramas do adsorvente colocados em contato com $25 \mathrm{~mL}$ de solução sintética contaminada $\left(20 \mathrm{mg} \mathrm{L}^{-1}\right)$ em $\mathrm{pH} 7$ (sem ajuste). As amostras foram mantidas em frascos plásticos vedados, sob agitação de $200 \mathrm{rpm}$ e temperatura de $25 \pm 2,0$ ${ }^{\circ} \mathrm{C}$ em incubadora refrigerada com agitação, modelo Tecnal (TE-421). Os ensaios foram realizados em duplicata e retirados em intervalos de tempo de até 2 horas. As amostras foram filtradas em membranas de celulose a $0,45 \mu \mathrm{m}$ e quantificadas em CLAE, conforme metodologia descrita (item 2.2). 
As quantidades adsorvidas em cada tempo foram determinadas conforme a Equação 1 .

$$
q(t)=\frac{\left(C_{o}-C(t)\right) * V}{m}
$$

Em que, $\mathrm{C}_{\mathrm{o}}$ é a concentração inicial de analito $\left(\mathrm{mg} \mathrm{L}^{-1}\right) ;(\mathrm{C}(\mathrm{t}))$ é a concentração remanescente de analito no tempo $(\mathrm{t})\left(\mathrm{mg} \mathrm{L}^{-1}\right)$ e $q(t)$ é a quantidade adsorvida de analito no tempo (t) $\left(\mathrm{mg} \mathrm{g}^{-1}\right)$; $\mathrm{m}$ é a massa de adsorvente (g) em base seca, e V é o volume da solução de analito em contato com o adsorvente (L).

As cinéticas de adsorção foram ajustadas conforme os modelos de pseudoprimeira e segunda ordem, dadas nas Equações 2 e 3.

A Equação 2 representa o Modelo de pseudo-primeira ordem (LAGERGREN, 1898):

$$
q(t)=q_{e q}\left(1-e^{-k_{1} t}\right)
$$

A Equação 3 representa o Modelo de pseudo-segunda ordem (HO e MCKAY, 1998):

$q(t)=q_{e q} \frac{q_{e q} k_{2} t}{q_{e q} k_{2} t+1}$

Em que, $q_{\text {eq }}$ representa a quantidade adsorvida no equilíbrio $\left(\mathrm{mg} \mathrm{g}^{-1}\right)$ e $\mathrm{k}_{1}$ e $\mathrm{k}_{2}$ são as constantes da taxa de adsorção de pseudoprimeira-ordem e pseudo-segunda ordem, respectivamente $\left(\mathrm{mg} \mathrm{g}^{-1} \mathrm{~min}^{-1}\right)$.

Os parâmetros dos modelos foram obtidos usando o software Statistica Windows $^{\circledR}$, versão 7.0. E para o método nãolinear simplex usou-se o ajuste de mínimos quadrados com função erro absoluto.

\subsubsection{Isotermas de adsorção}

Os ensaios para a obtenção das isotermas de adsorção foram realizados utilizando massas variando de 0,025 a $0,3 \mathrm{~g}$ postas em contato com $25 \mathrm{~mL}$ de solução sintética contaminada $\left(20 \mathrm{mg} \mathrm{L}^{-1}\right)$ em $\mathrm{pH}$ 7,5. As amostras foram mantidas durante 3 horas em incubadora com agitação orbital de 200 rpm e temperatura controlada de $25^{\circ} \mathrm{C}, 35^{\circ} \mathrm{C}$ e $45^{\circ} \mathrm{C}$. As amostras foram filtradas em membrana de celulose $0,45 \mu \mathrm{m}$ e quantificadas conforme metodologia descrita (item 2.2).

As quantidades adsorvidas nos equilíbrios foram determinadas pela Equação 4.

$$
q_{e q}=\frac{\left(C_{o}-C_{e q}\right) * V}{m}
$$

Em que, $\mathrm{C}_{\mathrm{eq}}$ é a concentração remanescente do analito no equilíbrio $\left(\mathrm{mg} \mathrm{L}^{-}\right.$ $\left.{ }^{1}\right)$.

As isotermas de adsorção foram ajustadas para os modelos de Langmuir e Freundlich conforme as Equações 5 e 6.

A equação 3 representa o modelo de Langmuir (1918) que é dada por:

$$
q_{e q}=\frac{q_{\max } K_{L} C_{e q}}{1+K_{L} C_{e q}}
$$

Em que, $\mathrm{K}_{\mathrm{L}}$ representa a constante de afinidade de Langmuir, $\mathrm{q}_{\text {máx }}$ é a capacidade máxima de adsorção de uma monocamada $\left(\mathrm{mg} \mathrm{g}^{-1}\right)$.

A equação 6 representa o modelo de Freundlich (1906) que é dado por:

$$
q_{e q}=K_{F} \cdot C_{e q}^{1 / n}
$$

Em que, $\mathrm{K}_{\mathrm{F}}$ representa a constante de capacidade de adsorção em multicamadas segundo o modelo de Freundlich e 1/n indica a existência de afinidade entre o diuron e o CAG, de modo que se seu valor for menor que 1, então a adsorção é favorável. 
Os parâmetros dos modelos foram obtidos usando o software Statistica para Windows ${ }^{\circledR}$, versão 7.0. E para o método nãolinear simplex usou-se o ajuste de mínimos quadrados com função erro absoluto.

\section{RESULTADOS E DISCUSSÕES}

\subsection{Caracterizações do CAG}

O CAG de coco de babaçu utilizado como adsorvente do presente estudo foi caracterizado segundo dois métodos. Primeiramente quanto ao PCZ que confere o comportamento elétrico superficial das partículas do adsorvente. E segundo, pelo método de BET e BJH que conferem ao adsorvente, dados de área específica superficial e o volume específico de poros, respectivamente, determinando-se características de macro, meso ou microporos ao adsorvente utilizado.

Assim, a Figura 1 abaixo apresenta o comportamento do $\mathrm{pH}$ em 11 pontos (PCZ) onde é determinado qual o valor de $\mathrm{pH}$ em que as cargas superficiais se anulam (REGALBUTO e ROBLES, 2004).

Figura 1 - Determinação do ponto de carga zero.

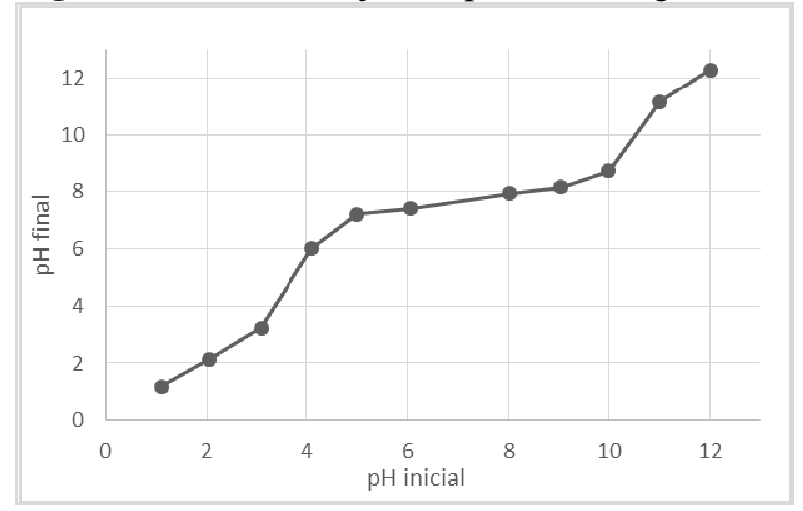

Como a dissociação dos grupos funcionais é dependente do $\mathrm{pH}$ da solução, se este estiver acima do PCZ do material adsorvente, então a superfície deste apresentará cargas predominantemente negativas em carboxilas e hidroxilas dissociadas. E se estiver abaixo, apresentará o efeito inverso.

Para tanto, é possível verificar na Figura 1 que a superfície do CAG não se caracteriza por grupos ácidos fortes como os carboxílicos, nem por bases fortes como os hidróxidos. A faixa do $\mathrm{pH}$ final que se mantém constante ocorre entre 5 e 9, apresentando o $\mathrm{pH}_{\mathrm{pcz}}$ de 7,58, o que evidencia que a superfície do CAG apresenta características que podem ser consideradas parcialmente neutras (GUILARDUCI et al., 2006. O pH utilizado nos ensaios foi de 7,5 estando coerente ao pHpcz.

A análise textural do CAG compreendeu o método de BET apresentado na Tabela 1.

Tabela 1 - Parâmetros texturais obtidos da análise de BET do CAG.

\begin{tabular}{lcc}
\hline & $\begin{array}{l}\text { Método curva t } \\
\text { - Microporos }\end{array}$ & $\begin{array}{l}\text { Método } \\
\text { BJH - } \\
\text { Mesoporos }\end{array}$ \\
\hline $\begin{array}{l}\mathrm{S}_{\mathrm{BET}} \\
\left(\mathrm{mg}^{2} \mathrm{~g}^{1}\right)\end{array}$ & 517,4 & 20,38 \\
\hline $\mathrm{S}\left(\mathrm{mg}^{2} \mathrm{~g}^{-1}\right)$ & 385 & 0,029 \\
$\mathrm{~V}\left(\mathrm{~cm}^{3} \mathrm{~g}^{-1}\right)$ & 0,276 & 34,12 \\
$\mathrm{~d}(\AA)$ & 3,66 & \\
\hline
\end{tabular}

O uso de carvão ativado granular ou em pó é amplamente utilizado em tratamento de água para remoção de micropoluentes orgânicos, porque apresenta extensa área superficial interna e estrutura de poros favorável (PELEKANI e SNOEYINK, 1999).

De acordo com os resultados da Tabela 1 foi possível verificar que o CAG apresenta uma área específica superficial elevada de $517,4\left(\mathrm{mg}^{2} \mathrm{~g}^{-1}\right)$, com $385 \mathrm{mg}^{2} \mathrm{~g}^{-1}$ de área de microporos. Segundo Teixeira et al. (2001), quanto maior for essa área de microporos, maior será a capacidade de adsorção para 
moléculas de dimensões pequenas. Assim, o uso deste CAG de coco de babaçu pode ser considerado muito favorável na remoção do diuron. Entretanto, nota-se uma baixa presença no desenvolvimento de mesoporos $\left(20,38 \mathrm{mg}^{2} \mathrm{~g}^{-1}\right)$.

\subsection{Cinética de adsorção}

A cinética de adsorção do diuron em CAG de coco de babaçu, bem como os ajustes dos dados experimentais dos modelos cinéticos de pseudo-primeira e pseudosegunda ordens são apresentados na Figura 2.

Figura 2 - Cinética de adsorção (dosagem biomassa $=20 \mathrm{~g} \mathrm{~L}^{-1}, 200 \mathrm{rpm}, 25^{\circ} \mathrm{C}, 2$ horas, $\mathrm{C}_{\mathrm{o}}=20 \mathrm{mg} \mathrm{L}^{1}$ ).

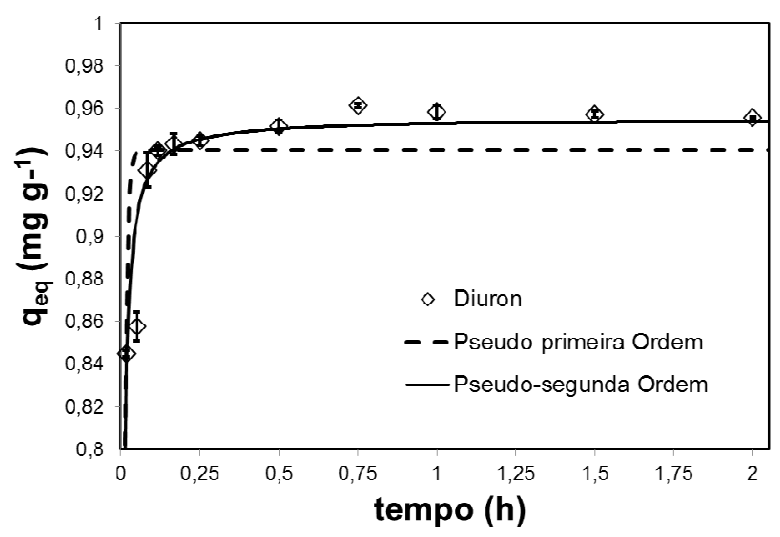

A Figura 2 apresenta um bom resultado quanto ao equilíbrio de adsorção alcançado em aproximadamente 30 minutos um $\mathrm{q}_{\mathrm{eq}} \mathrm{de}$ $0,952 \mathrm{mg} \mathrm{g}^{-1}\left(25^{\circ} \mathrm{C}\right)$. Esse tempo de contato foi o mesmo ao obtido nos estudos de Bahri et al. (2012), que apresentou em 30 minutos uma remoção de $14,266 \mathrm{mg} \mathrm{g}^{-1}\left(25^{\circ} \mathrm{C}\right)$ para o diuron em sementes de uvas com tratamento térmico $\left(350-550^{\circ} \mathrm{C}\right)$ e químico $(5 \%$ $\left.\mathrm{H}_{2} \mathrm{SO}_{4}\right)$ e ativação em $\mathrm{H}_{3} \mathrm{PO}_{4}$. Observou-se que a melhora da quantidade adsorvida $\left(\mathrm{q}_{\mathrm{eq}}\right)$ se deu devido a elevada área superficial (1139 $\mathrm{mg}^{2} \mathrm{~g}^{-1}$ ) da semente de uva em comparação ao do CAG de coco de babaçu $\left(517,4 \mathrm{mg}^{2} \mathrm{~g}^{-1}\right)$, bem como o mesmo ter passado por um tratamento químico e ativação, fato este não ocorrido para CAG de coco de babaçu.

Apesar dos estudos apresentarem maiores quantidades adsorvidas para a semente de uva, ressalta-se a dificuldade de se obter grandes quantidades desta biomassa em comparação ao subproduto coco de babaçu. O coco de babaçu é gerado em grandes quantidades, sendo a fabricação deste adsorvente viável economicamente.

Os resultados obtidos para os parâmetros dos modelos pseudo-primeira e pseudo-segunda ordem são apresentados na Tabela 2.

Tabela 2 - Modelos cinéticos de pseudoprimeira e pseudo-segunda ordem.

\begin{tabular}{|c|c|c|}
\hline Parâmetros & $\begin{array}{l}\text { Pseudo- } \\
\text { Primeira } \\
\text { Ordem }\end{array}$ & $\begin{array}{l}\text { Pseudo- } \\
\text { Segunda } \\
\text { Ordem }\end{array}$ \\
\hline $\mathrm{q}_{\text {eq experimental }}\left(\mathrm{mg} \mathrm{g}^{-1}\right)$ & $0,952 \pm 0,003$ & \\
\hline qeq calculado & 0,941 & 0,955 \\
\hline $\mathrm{k}\left(\mathrm{mg} \mathrm{g}^{-1} \min ^{-1}\right)$ & 135,489 & 406,305 \\
\hline $\mathrm{R}^{2}$ & 0,995 & 0,998 \\
\hline
\end{tabular}

De acordo com a Tabela 2, juntamente com a Figura 2, o modelo de pseudo-segunda ordem representou melhor os dados da cinética de adsorção do diuron. Foi verificado um $\mathrm{q}_{\mathrm{eq}}$ calculado $\left(0,955 \mathrm{mg} \mathrm{g}^{-1}\right)$ próximo ao obtido experimentalmente $\left(0,952 \mathrm{mg} \mathrm{g}^{-1}\right)$, bem como um melhor coeficiente de correlação $(0,998)$. Este modelo pressupõe que a etapa limitante para a adsorção envolve o número de sítios disponíveis para a adsorção (MIMURA et al., 2010). Os dados experimentais obtidos por Bahri et al. (2012) também foram bem ajustados para o modelo de pseudo-segunda ordem, comportamento análogo ao presente estudo.

\subsection{Isotermas de adsorção}

Os dados de equilíbrio, conhecido como isotermas de adsorção são requisitos básicos para avaliar sistemas de adsorção. 
Os dados experimentais de equilíbrio foram ajustados segundo os modelos de Langmuir e Freundlich, apresentados na Figura 3.

Figura 3 - Ajuste dos dados experimentais quanto aos modelos de Langmuir e Freundlich. Isotermas de adsorção (a) $\mathrm{T}=25^{\circ} \mathrm{C}$; (b) $\mathrm{T}=35^{\circ} \mathrm{C}$; (c) $\mathrm{T}=45^{\circ} \mathrm{C}$.

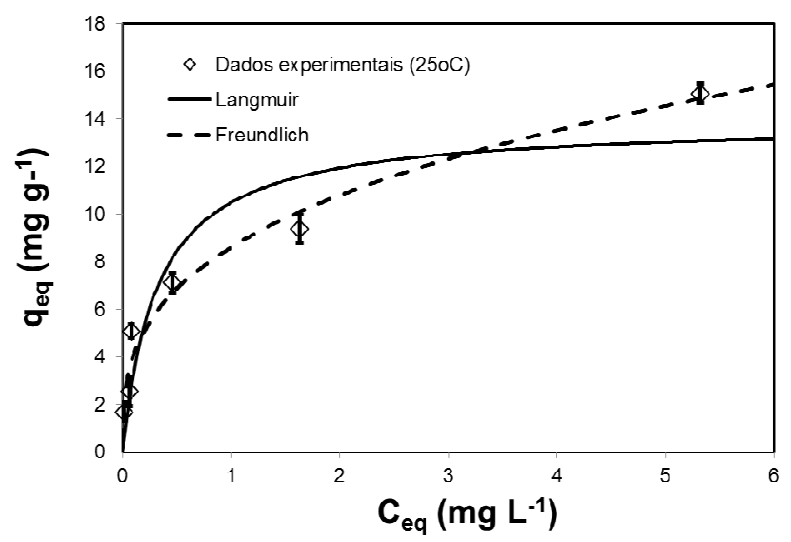

(a)

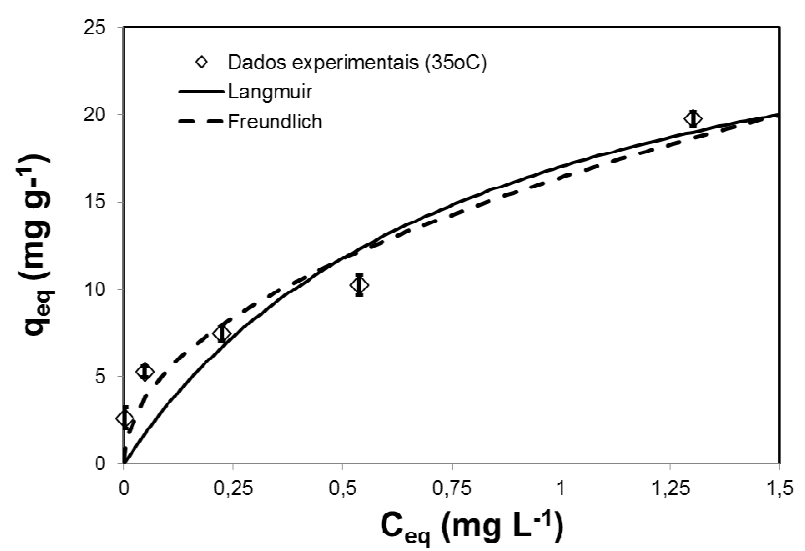

(b)

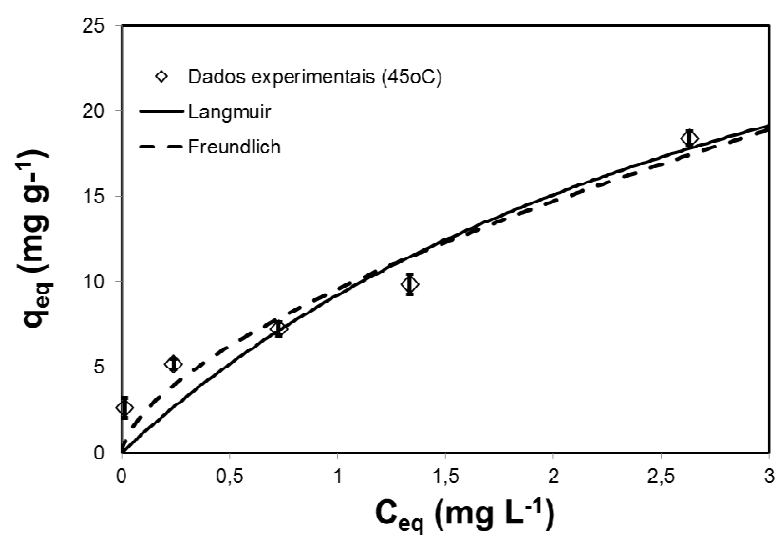

(c)

Os resultados obtidos para os parâmetros desses modelos são apresentados na Tabela 3.

Tabela 3 - Parâmetros dos modelos de Langmuir e Freundlich.

\begin{tabular}{lcc}
\hline Parâmetros & Langmuir & Freundlich \\
\hline Temperatura & $25^{\circ} \mathrm{C}$ & \\
\hline $\mathrm{q}_{\text {máx }}\left(\mathrm{mg} \mathrm{g}^{-1}\right)$ & 13,849 & - \\
$\mathrm{K}$ & 3,113 & 8,602 \\
$\mathrm{R}^{2}$ & 0,930 & 0,986 \\
$\mathrm{n}$ & - & 3,0660 \\
\hline Temperatura & $35^{\circ} \mathrm{C}$ & \\
\hline $\mathrm{q}_{\text {máx }}\left(\mathrm{mg} \mathrm{g}^{-1}\right)$ & 30,827 & - \\
$\mathrm{K}$ & 1,233 & 16,405 \\
$\mathrm{R}^{2}$ & 0,927 & 0,968 \\
$\mathrm{n}$ & - & 2,060 \\
\hline Temperatura & $45^{\circ} \mathrm{C}$ & \\
\hline $\mathrm{q}_{\text {máx }}\left(\mathrm{mg} \mathrm{g}^{-1}\right)$ & 41,432 & - \\
$\mathrm{K}$ & 0,287 & 9,581 \\
$\mathrm{R}^{2}$ & 0,946 & 0,968 \\
$\mathrm{n}$ & - & 1,615 \\
\hline
\end{tabular}

Mediante análises da Figura 3 e os parâmetros apresentados na Tabela 3 , é possível verificar que nas faixas de concentração de diuron e massa de adsorvente estudadas, a isoterma de Freundlich representou melhor os dados obtidos nas três temperaturas. Isso demonstra que há uma diminuição da energia de adsorção à medida 
que ocorre o recobrimento da superfície pelo adsorvato, o que caracteriza presença de superfícies heterogêneas.

$\mathrm{O}$ parâmetro $\mathrm{K}_{\mathrm{F}}$ isoterma de Freundlich indica a capacidade do adsorvente em reter $\mathrm{o}$ adsorvato ( $\mathrm{mg} \mathrm{g}^{-1}$ ) (SPOSITO, 1980).

O parâmetro (n) está relacionado com a a distribuição dos sítios energéticos de do adsorvente, ou seja, quanto maior o valor de $\mathrm{n}$, maior a heterogeneidade dos sítios de adsorção (SPOSITO, 1980). Comparando os resultados obtidos na Tabela 3, para o parâmetro (n), é possível verificar que a adsorção foi mais favorável em $25^{\circ} \mathrm{C}$ com $1 / \mathrm{n}$ correspondendo a 0,326 em comparação às demais temperaturas, com 0,485 e 0,619, respectivamente. Os valores de $\mathrm{n}$ obtidos demonstram a heterogeneidade do material adsorvente e indica sítios altamente energéticos. O coeficiente de determinação das isotermas de Freundlich também apresentaram $\mathrm{R}^{2}=0,986$ para $25^{\circ} \mathrm{C}$, sendo superior as demais temperaturas.

Dentro das condições trabalhadas, os ajustes utilizados nas isotermas de Langmuir não foram satisfatórios. Porém, a análise dos parâmetros $\mathrm{K}_{\mathrm{L}}$ demonstraram que a afinidade do adsorvente diminui com o aumento da temperatura. Porém, a capacidade máxima aumentou com a temperatura. Estes resultados indicam a necessidade de mais ensaios para confirmar o comportamento do processo de adsorção de diuron no carvão utilizado.

\section{CONCLUSÃO}

O uso de herbicidas altamente persistentes no ambiente, como o diuron, está diretamente relacionado a uma elevada contaminação ambiental.

O presente estudo apresentou que é possível removê-lo de águas sintéticas com CAG de coco de babaçu. A adsorção demonstrou um tempo de contato de 30 minutos. A cinética foi melhor representada pelo modelo de pseudo-segunda ordem.
No estudo de equilíbrio as isotermas foram melhor representadas pelo modelo de Freundlich, de modo que em $45^{\circ} \mathrm{C}$ obteve o maior valor de $\mathrm{q}_{\text {máx }}$ atingindo $41,432 \mathrm{mg} \mathrm{g}^{-1}$.

\section{NOMENCLATURA}

\begin{tabular}{|c|c|}
\hline BET & Brunauer-Emmett-Teller \\
\hline BJH & Barret-Joyner-Halenda \\
\hline $\mathrm{C}_{\mathrm{o}}$ & $\begin{array}{l}\text { Concentração inicial de analito } \\
\left(\mathrm{mg} \mathrm{L}^{-1}\right)\end{array}$ \\
\hline $\mathrm{C}_{\mathrm{eq}}$ & $\begin{array}{l}\text { Concentração remanescente do } \\
\text { analito no equilíbrio }\left(\mathrm{mg} \mathrm{L}^{-1}\right)\end{array}$ \\
\hline $\mathrm{C}(\mathrm{t})$ & $\begin{array}{l}\text { Concentração remanescente de } \\
\text { analito no tempo }(\mathrm{t})\left(\mathrm{mg} \mathrm{L}^{-1}\right)\end{array}$ \\
\hline CAG & $\begin{array}{l}\text { Carvão ativado granular de } \\
\text { coco de babaçu }\end{array}$ \\
\hline CLAE & $\begin{array}{l}\text { Cromatografia Líquida de Alta } \\
\text { Eficiência }\end{array}$ \\
\hline$d_{\text {meso }}$ & $\begin{array}{l}\text { Diâmetro do poro pelo método } \\
\text { BJH }\end{array}$ \\
\hline$d_{\text {micro }}$ & $\begin{array}{l}\text { Diâmetro do poro pelo método } \\
\text { DA }\end{array}$ \\
\hline $\mathrm{k}_{1}$ & $\begin{array}{l}\text { Constante da taxa de adsorção } \\
\text { do modelo pseudo-primeira } \\
\text { ordem }\left(\mathrm{min}^{-1}\right)\end{array}$ \\
\hline $\mathrm{k}_{2}$ & $\begin{array}{l}\text { Constante da taxa de adsorção } \\
\text { do modelo pseudo-segunda } \\
\text { ordem }\left(\mathrm{min}^{-1}\right)\end{array}$ \\
\hline $\mathrm{K}_{\mathrm{L}}$ & Constante de Langmuir \\
\hline $\mathrm{K}_{\mathrm{F}}$ & $\begin{array}{l}\text { Constante de capacidade de } \\
\text { adsorção de Freundlick }\end{array}$ \\
\hline M & $\begin{array}{l}\text { Massa de adsorvente em base } \\
\text { seca }(g)\end{array}$ \\
\hline & pH no ponto de carga zero \\
\hline $\mathrm{q}_{\text {eq }}$ & $\begin{array}{l}\text { Quantidade adsorvida de analito } \\
\text { no equilíbrio }\left(\mathrm{mg} \mathrm{g}^{-1}\right)\end{array}$ \\
\hline$q(t)$ & $\begin{array}{l}\text { Quantidade adsorvida de analito } \\
\text { no tempo }(\mathrm{t})\left(\mathrm{mg} \mathrm{g}^{-1}\right)\end{array}$ \\
\hline $\mathrm{R}^{2}$ & Coeficiente de determinação \\
\hline $\mathrm{S}_{\mathrm{BET}}$ & Área superficial específica BET \\
\hline$S_{\text {meso }}$ & $\begin{array}{l}\text { Área de mesoporos pelo método } \\
\text { BJH }\end{array}$ \\
\hline & Área de microporos pelo \\
\hline
\end{tabular}




$\begin{array}{ll}\text { SINDIVEG } & \begin{array}{l}\text { método " } \mathrm{t} \text { " } \\ \text { Sindicato Nacional da Indústria } \\ \text { de Produtos para Defesa } \\ \text { Vegetal } \\ \text { Volume da solução de analito } \\ \text { em contato com o adsorvente } \\ \text { (L) }\end{array} \\ & \begin{array}{l}\text { Volume do poro pelo método } \\ \text { BJH }\end{array} \\ & \begin{array}{l}\text { Volume do poro pelo método } \\ \text { "teso }\end{array}\end{array}$

REFERÊNCIAS

BAHRI, M. A.; CALVO, L.; GILARRANZ, M. A.; RODRIGUES, J. J. Activated carbon from grape seeds upon chemical activation with phosphoric acid: Application to the adsorption of diuron from water. Chemical Engineering Journal, v. 203, p. 348-356, 2012.

BHATNAGAR, A.; SILLANPÄÄ, M. Utilization of agro-industrial and municipal waste materials as potential adsorbents for water treatment - A review. Chemical Engineering Journal, v. 157, p. 277-296, 2010.

BRITO, N. M.; AMARANTE JR., O. P.; ABAKERLI, R.; SANTOS, T. C. R. Risco de contaminação de águas por pesticidas aplicados em plantações de eucaliptos e coqueiros: Análise Preliminar. Pesticidas: Revista de Ecotoxicologia e Meio Ambiente, v. 11, p. 93104, 2001.

DI BERNARDO, L.; DANTAS, A.D.B. Métodos e técnicas de tratamento de água. 2. ed. São Carlos: RiMa, 2005. v. 2.

DI BERNARDO DANTAS, A.; PASCHOALATO, C. F. R. EQUIPE; MARTINEZ, M. S.; DI BERNARDO, L. A remoção do diuron e hexazinona de águas subterrâneas do aquífero Guarani. Revista Brasileira de Engenharia Química, v. 28, n. 3, 2011.
FIGUEIREDO, J. L.; RIBEIRO, F. R. Catálise heterogênea. Lisboa, Fundação Calouste Gulbenkian, 1987.

FREUNDLICH, H. M. F. Uber die adsorption in losungen. Zeitschrift fur Phusikalische Chemie (Leipzig), v. 57ª p. 385-470, 1906.

GUILARDUCI, V. V. S.; MESQUITA, J. P.; MARTELLI, P. B.; GORGULHO, H. F. Adsorção de fenol em carvão ativado em meio alcalino. Química Nova, v. 29, n. 6, p. 12261232, 2006.

GRÜTZMACHER, D. D.; GRÜTZMACHER, A. D.; AGOSTINETTO, D.; LOECK, A. E.; ROMAN, R.; PEIXOTO, S. C.; ZANELLA, R. Monitoramento de agrotóxicos em dois mananciais hídricos no Sul do Brasil. Revista Brasileira de Engenharia Agrícola e Ambiental, v. 12, n. 6, p. 632-637, 2008.

HO, Y.S.; MCKAY, G. Kinetic models for the sorption of dye from aqueous solution by wood. Trans IChemE, v. 76B, p. 183-191, 1998.

KONSTANTINOU, I. K.; HELA, D. G.; ALBANIS, T. A. The status of pesticide pollution in surface waters (rivers and lakes) of Greece. Part I. Review on occurrence and levels. Environmental Pollution, v. 141, n. 03, p. 555-570, 2006.

LAGERGREN, S. Zur theorie der sogenannten adsorption gelösterstoffe. Bihang TillKungligaSvenskaVetenskaps-akademiens.

Handlingar, Band, 24, afd.II, n.4, p. 1-39, 1898.

LANGMUIR, I. The adsorption of gases on plane surfaces of glass, mica and platinum. Journal of the American Chemical Society, v.40, p.1361-1403, 1918.

LAVORENTI, A.; LANÇAS, F. M. Monitoramento de hexazinone e diuron em águas de superfície (rios) em regiões de canaviais do Estado de São Paulo. DuPont Agricultura e Nutrição. Paulínia, SP, 52 p., 2003. 
MIMURA, A.M.S.; VIEIRA, T.V.A.; MARTELLI, P.B.; GORGULHO, H.F. Aplicação da casca de arroz na adsorção dos íons $\mathrm{Cu}^{2+}, \mathrm{Al}^{3+}, \mathrm{Ni}^{2+}$ e $\mathrm{Zn}^{2+}$. Química Nova, v. 33, n. 6, p.1279-1284, 2010.

NETO, M. L. F.; SARCINELLI, P. N. Agrotóxicos em água potável para consumo humano: uma abordagem de avaliação de risco e contribuição ao processo de utilização da legislação brasileira. Eng. Sanit. Ambient., v. 14, n.1, p. 69-78, 2009.

PELEKANI, C. e SNOEYINK, V. L. Adsorção competitiva em água potável: papel do tamanho do poro ativado de carbono. Água Res., v. 33, n. 5, p. 1209-1219, 1999.

PIZA, A.V.T. Estudo da capacidade de adsorção dos herbicidas diuron e hexazinona em carvões ativados. 185 f. Dissertação (Mestrado em Tecnologia Ambiental) - Centro de Ciências Exatas, Naturais e Tecnologias, UNAERP, Ribeirão Preto, São Paulo, 2008.

REGAlbutO, J. R.; ROBLES, J. O. The engineering of Pt/carbon catalyst preparation for application on proton exchange fuel cell membrane (PEFCM). Catalysis Laboratory, University of Illinois at Chicago. 2004

RUEDA, R.B. Pré-oxidação e adsorção em carvão ativado granular para remoção de herbicidas diuron e hexazinona de águas subterrâneas. 175 f. Dissertação (Mestrado em Tecnologia Ambiental) - Centro de Ciências Exatas, Naturais e Tecnologias, UNAERP, Ribeirão Preto, São Paulo, 2008.

SANCHES, S. M.; da SILVA, C. H. T. P.; de CAMPOS, S. X.; VIEIRA, E. M. Pesticidas e seus respectivos riscos associados a contaminação da água. Pesticidas: Revista de Ecotoxicologia e Meio Ambiente, v. 13, p. 5358, 2003.

SCHWARZENBACH, R.P.; GIGER, W.; HOEHN, E.; SCHNEIDER, J. K. Behaviour of organic compounds during infiltration of river water to groundwater: field studies.
Enviromental Science and Techonology, v. 17, n. 8, p. 472-479, 2006.

SINDIVEG - SINDICATO NACIONAL DA INSÚSTRIA DE PRODUTOS PARA DEFESA VEGETAL. Vendas de defensivos agrícolas por culturas de destinação e classes - 2011 a 2013. Disponível em: < http://www.sindiveg.org.br/estatisticas.php>. Acesso em nov. 2014.

SILVA, C. L. d. Análise da vulnerabilidade ambiental aos principais pesticidas recomendados para os sistemas de produção de algodão, arroz, café, cana-de-açúcar, citros, milho e soja. 135 f. Dissertação (Mestrado em Engenharia Agrícola) Faculdade de Engenharia Agrícola, Unicamp, Campinas, São Paulo, 2004.

SPOSITO, G. Derivation of the Freundlich Equation for Ion Exchange Reactions in Soils. Soil Science Society y America Journal, v. 44, p. 652-654, 1980.

TEIXEIRA, A. G. V.; COUTINHO, F. M. B.; GOMES, A. S. Principais métodos de caracterização da porosidade de resinas a base de divinilbenzeno. Química Nova, v. 24, n. 6, p. 808-818, 2001.

TERRA, F. H. B.; V. M. PELAEZ. A evolução da indústria de agrotóxicos no Brasil de 2001 a 2007: a expansão da agricultura e as modificações na lei de agrotóxicos. 46th Congress, Rio Branco, Acre, Brasil, Sociedade Brasileira de Economia, Administracao e Sociologia Rural (SOBER), 2008.

ZOLGHARNEIN, J., A. SHAHMORADI, A.; GHASEMI, J. Pesticides Removal Using Conventional and Low-Cost Adsorbents: A Review. Clean-Soil, Air, Water, v. 39, n.12, p. 1105-1119, 2011.

\section{AGRADECIMENTOS}

O presente trabalho foi realizado com o apoio da Coordenação de Aperfeiçoamento de Pessoal de Nível Superior - CAPES - BR. 